\title{
Due note critiche ai Commentarii linguae Graecae di Guillaume Budé
}

\author{
GIANMARIO CATTANEO
}

This paper proves that a quotation Budé ascribed to "Theodore Gaza's Laelius" was actually taken from Gaza's Greek translation of Cicero's Cato maior de senectute. Moreover, Budé cites an excerpt from George Scholarios' Confessio fidei posterior, a work with which Budé got acquainted thanks to his teacher George Hermonymos, who translated it into Latin. ${ }^{*}$

Il 2018 sarà ricordato come un anno ricchissimo per quanto riguarda gli studi e gli eventi dedicati a Guillaume Budé (1468-1540). ${ }^{1}$ Infatti, è stata pubblicata l'edizione con traduzione francese dei primi tre libri del $D e$ asse curata da Luigi-Alberto Sanchi $^{2}$ e si è tenuto a Parigi un convegno internazionale dal titolo Les Noces de Philologie et de Guillaume Budé in occasione del $550^{\circ}$ anniversario dalla nascita. Inoltre, è uscito un importante contributo dello stesso Sanchi dedicato alla biblioteca intellettuale di Budé. ${ }^{3}$ Sulla base delle citazioni di autori classici e medievali presenti nei Commentarii linguae Graecae, ${ }^{4}$ nel De asse, ${ }^{5}$ nelle Annotationes in Pandectas ${ }^{6}$ e negli inediti carnets autografi di

* Ringrazio Luigi-Alberto Sanchi e i due anonimi referee di Humanistica Lovaniensia per la lettura e i preziosi suggerimenti.

${ }^{1}$ Sulla vita e le opere di Budé rimando ad alcuni dei principali studi usciti nell'ultimo secolo: L. Delaruelle, Guillaume Budé. Les origines, les débuts, les idées maîtresses (Paris, 1907; rist. Genève, 2012); D.O. McNeil, Guillaume Budé and Humanism in the Reign of Francis I (Genève, 1975); M.-M. de La Garanderie, Christianisme et lettres profanes. Essai sur l'humanisme français (1515-1535) et sur la pensée de Guillaume Budé (Paris, 1995); J.-F. Maillard, M. Portalier et al., Guillaume Budé, in Iid., La France des humanistes. Hellénistes I (Turnhout, 1999), 41-96; M.-M. de La Garanderie, Guillaume Budé, philosophe de la culture (Paris, 2010).

${ }^{2}$ L.-A. Sanchi (ed.), Guillaume Budé, De asse et partibus eius. L'as et ses fractions. Livres I-III (Genève, 2018).

${ }^{3}$ L.-A. Sanchi, "La bibliothèque de Guillaume Budé", Arts et Savoirs, 10 (2018), doi: 10.4000/aes. 1273 .

${ }^{4}$ Sui Commentarii si veda L.-A. Sanchi, Les Commentaires de la langue grecque de Guillaume Budé. L'oeuvre, ses sources, sa préparation (Genève, 2006). I Commentarii conobbero un'edizione licenziata da Budé nel 1529 e una postuma nel 1548, poi variamente ristampata; sulle due edizioni si veda in particolare Sanchi 2006 (come a n. 4), 39-48.

${ }^{5}$ Sul De asse e le sue diverse edizioni si veda Sanchi 2018 (come a n. 2), xviii-xxviii.

${ }^{6}$ Pubblicate nel 1508, 1516 e infine nel 1535. Si veda Sanchi 2018 (come a n. 3), n. 4. 
Budé, ${ }^{7}$ Sanchi ha stilato una lista degli autori letti da Budé, per i quali sono stati identificati quarantasei manoscritti e stampe posseduti o postillati da Budé.

1. Nel suo repertorio Sanchi ha il merito di mettere in luce alcuni loci critici all'interno della "biblioteca" di Budé e così aprire la strada a nuove riflessioni sui libri consultati dall'umanista francese. Un esempio si trova al numero 191 della lista:

191. Gaza, Théodore, trad. grecque de Cicéron, Laelius: Comm. ${ }^{8}$

Il brano dei Commentarii in cui Budé cita la traduzione di Teodoro Gaza è il seguente:

$\Delta$ tá

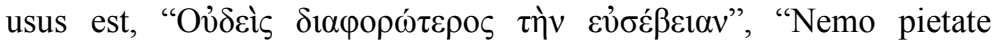
praestantior". 9

L'aggettivo "insigne" viene espresso da Platone con la parola $\delta$ ió $\varphi$ o $\varsigma_{,}{ }^{10}$ che usò anche Gaza nel Lelio di Cicerone: "Nessuno più

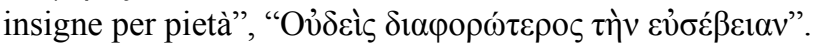

Secondo Budé, Teodoro Gaza (1410 ca.-1475)11 tradusse la frase "Nemo

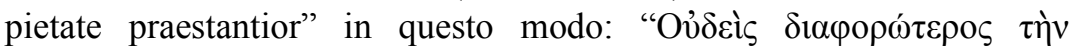
$\varepsilon v ่ \sigma \varepsilon ́ \beta \varepsilon 1 \alpha v "$, ed essa sarebbe presente nel Laelius de amicitia di Cicerone. Questa citazione presenta però due ordini di problemi.

Innanzitutto, la frase latina tradotta da Gaza non è contenuta nel Laelius, bensì nel Cato maior de senectute (84):

\footnotetext{
${ }^{7} \mathrm{Su}$ questi carnets, che sono conservati in una collezione privata a Ginevra e ammontano a circa 1800 fogli, si veda il quadro tracciato da Sanchi 2006 (come a n. 4), 142-146, con bibliografia precedente.

${ }^{8}$ Sanchi 2018 (come a n. 3), 10.

${ }^{9} \mathrm{Il}$ passo si legge in G. Budé, Commentarii linguae Graecae (Parisiis, 1529 [USTC 146015]), 353; Id., Commentarii linguae Graecae (Parisiis, 1548 [USTC 149947]), 410; Id., Commentarii linguae Graecae (Basileae, 1556 [USTC 623527]), 566.

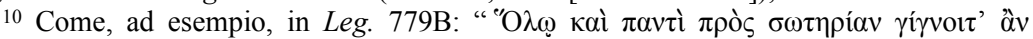

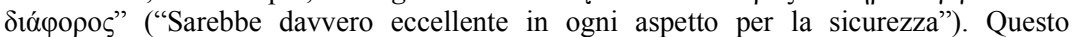
aggettivo ha come significato principale quello di "diverso", "differente". Si veda a tal

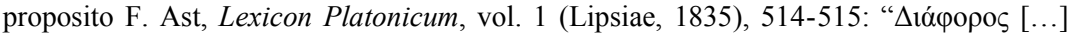
praestans, insignis".

${ }^{11}$ Sulla vita e le opere di Gaza rimando a C. Bianca, "Gaza, Teodoro", in Dizionario biografico degli Italiani, vol. 52 (Roma, 1999), 737-746.
} 
Proficiscar enim non ad eos solum viros de quibus ante dixi, verum etiam ad Catonem meum, quo nemo vir melior natus est, nemo pietate praestantior.

A parlare è Catone il Censore, che dice di desiderare la morte poiché così si potrà ricongiungere con il figlio Marco Porcio Catone Liciniano, morto nel 152 a.C.

Teodoro Gaza realizzò la traduzione del Cato maior de senectute tra il 1451 e il 1455, forse su impulso di papa Niccolò V (1397-1455), e continuò a rielaborarla negli anni successivi. ${ }^{12}$ Nella traduzione greca di Gaza, Cato 84 è reso in questo modo:

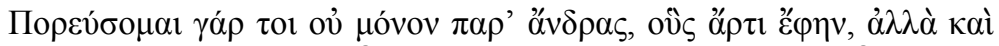

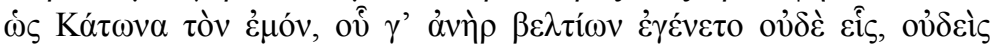

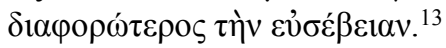

Il secondo problema è legato al fatto che non si è conservata nessuna traduzione in greco del De amicitia attribuita a Gaza. Teodoro è ricordato come autore di una traduzione del De amicitia da Raffaele Maffei da Volterra (1451-1522) nei Commentariorum rerum urbanarum libri, ${ }^{14}$ ma di questa versione non possediamo altre testimonianze né si è conservato alcun brano per via indiretta. ${ }^{15}$ L'unica traduzione in greco classico del De amicitia ad essere giunta fino a noi è quella di Denis

$12 \mathrm{Su}$ Gaza traduttore del De senectute si veda G. Salanitro (ed.), M. Tullii Ciceronis Liber de senectute in Graecum translatus (Leipzig, 1987). Più in generale, su Gaza traduttore si vedano la panoramica tracciata da Id., "Teodoro Gaza traduttore di testi classici”, in M. Cortesi, E.V. Maltese (ed.), Dotti bizantini e libri greci nell'Italia del secolo XV (Napoli, 1992), 223-228, e P. Beullens, A. Gotthlef, "Theodore Gaza's Translation of Aristotle's De animalibus: Content, Influence, and Date", Greek, Roman, and Byzantine Studies 47 (2007), 469-513.

13 Salanitro 1987 (come a n. 12), 60.

14 R. Maffei, Commentariorum urbanorum libri (Romae, 1506 [USTC 839511]), f. 308v: "Theodorus Gaza [...] convertit in Latinum sermonem Theophrastum De plantis et Aristotelis libros De animalibus et Problematum; praeterea libros Ciceronis De senectute et amicitia in Graecam elegantissime linguam."

${ }^{15}$ L'informazione fornita da Raffaele Maffei fu poi ripresa in repertori e opere erudite settecentesche, come in H. Hody, De Graecis illustribus linguae Graecae literarumque humaniorum instauratoribus (Londini, 1742), 67, e, in maniera indiretta, viene fatta propria anche da M. von Albrecht, Geschichte der römischen Literatur, vol. 1 (Berlin Boston, MA, 2012), 466: "Theodoros von Gaza übersetzt im 15. Jh. Ciceros Cato und Laelius ins Griechische.” 
Pétau (1583-1652), versione pubblicata a Parigi un anno dopo la morte del suo autore. ${ }^{16}$

Ritornando a Budé, egli dimostra di conoscere bene la traduzione di Gaza del Cato maior e la cita altre volte nei Commentarii: la traduzione viene sempre identificata in maniera corretta ("Gaza ex Cicerone De senectute" "Gaza [...] cum Catonem Ciceronis verteret", "Gaza in Catone", "Gaza [...] in libro De senectute vertendo", "Cicero in Catone $[\ldots]$ quod Gaza [...] dixit"), ${ }^{17}$ se si eccettua un caso in cui Budé si limita a dire "Gaza ex Cicerone". 18

Sorprende quindi l'erronea attribuzione al De amicitia, che potrebbe dipendere da vari fattori. Innanzitutto, siccome non esistono né manoscritti né stampe in cui la versione di Teodoro Gaza viene identificata con il titolo di Laelius, ${ }^{19}$ pare difficile che Budé lavorasse con il testo greco davanti agli occhi. Ė invece possibile che l'errore di Budé sia da ricondurre ai lavori preparatori per i Commentarii. ${ }^{20}$

Giacché uno dei personaggi cui Catone si rivolge nel De senectute è Gaio Lelio, durante la schedatura dell'opera Budé potrebbe avere trascritto la frase sotto un lemma del tipo "Gaza Laelio" ("Gaza a Lelio"), e questa imprecisione si sarebbe poi riverberata nei Commentarii. Oppure, dal momento che i Commentarii di Raffaele Maffei conobbero una grande fortuna editoriale e furono stampati anche a Parigi nel $1511,{ }^{21}$ Budé potrebbe essere stato influenzato dalla notizia data da Raffaele Maffei e, nei suoi appunti, aver confuso le traduzioni dei due dialoghi ciceroniani. O ancora, Budé potrebbe avere impropriamente attribuito al Cato maior il titolo di Laelius, dal nome di uno dei personaggi del dialogo. Tuttavia, questa ipotesi pare la meno plausibile, giacché in un'altra circostanza Budé utilizza correttamente il titolo Laelius per indicare il De amicitia: "Cicero in Laelio: 'Ditior enim videtur esse et affluentior vera amicitia'."’22

16 D. Pétau (tr.), M. Tullii Ciceronis Laelius sive de amicitia Graece redditus (Lutetiae Parisiorum, 1653).

${ }^{17}$ Le citazioni provengono rispettivamente da Budé 1548 (come a n. 9), 640, 643, 813, 922, 1052.

18 Budé 1548 (come a n. 9), 668.

${ }^{19}$ Si veda l'elenco dei testimoni della traduzione in Salanitro 1987 (come a n. 12), viii-xii.

${ }^{20}$ Non ci sono riferimenti a Gaza nei carnets autografi di Budé conservati a Ginevra, come si evince da Sanchi 2006 (come a n. 4), 237-259.

${ }^{21}$ R. Maffei, Commentariorum urbanorum octo et triginta libri (Parisiis, 1511 [USTC 143764]).

22 Budé 1548 (come a n. 9), 1077; Cic. Lael. 58. 
In conclusione, la citazione attribuita da Budé a "Gaza in Laelio Ciceronis" deve essere ricondotta alla traduzione del Cato maior. ${ }^{23}$ Come nota Sanchi, ${ }^{24}$ forse Budé lesse quest'opera nel manoscritto Paris, Bibliothèque nationale de France, ms. Gr. 2071,25 copiato dal suo maestro Giorgio Ermonimo (1430 ca.-1511 ca.). ${ }^{26}$

2. Tra gli autori greci citati da Budé è registrato anche Giorgio Scolario, patriarca ortodosso di Costantinopoli con il nome di Gennadio II (1400 ca.-1472 ca.):

\section{Scholarios, Georges, La Trinité: Comm., carnet VI. ${ }^{27}$}

Nei carnets autografi viene citato un passo di Gennadio sulla trinità

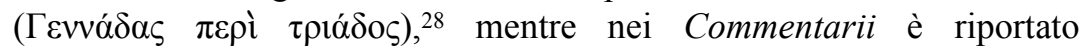
solamente il nome dell'autore:

$\Sigma \varepsilon \beta \alpha ́ \zeta o \mu \alpha \iota$ etiam "colere" et "venerari religiose" significat. Gennadas:

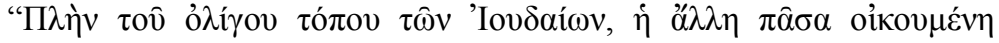

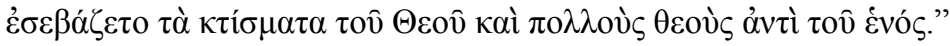

$\Sigma \varepsilon \beta \alpha ́ \zeta o \mu \alpha r$ significa anche "onorare" e "venerare devotamente". Gennadio: "Eccetto la piccola regione della Giudea, tutto il restante mondo venerava le creature di Dio e molti dei anziché l'unico Dio."

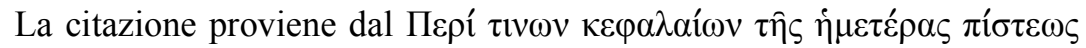
("Su qualche capitolo della nostra fede") di Scolario, noto anche con il titolo latino di Confessio fidei posterior (4): ${ }^{29}$

${ }^{23}$ L'esempio "Gaza in Laelio Ciceronis" è riportato anche nel Thesaurus Graecae linguae di Henri Estienne: H. Stephanus, Thesaurus Graecae linguae, vol. 3 (Parisiis, 1572), 97.

24 Sanchi 2018 (come a n. 3), 10: “190. Gaza, Théodore, trad. grecque de Cicéron, Caton l'Ancien: prob. ms. Paris, BnF, gr. 2071, copié par Hermonyme [première éd. Venise, Alde, et Florence, Giunta, 1517]."

${ }^{25} \mathrm{Su}$ questo manoscritto si veda J.-F. Maillard, J.-M. Flamand, George Hermonyme, in Iid., La France des Humanistes. Hellénistes II (Turnhout, 2010), 1-214 (a 83-84).

${ }^{26} \mathrm{Su}$ Ermonimo e la sua attività di professore e copista si vedano in particolare M.P. Kalatzi, Hermonymos. A Study in Scribal, Literary and Teaching Activities in the Fifteenth and Early Sixteenth Centuries (Athens, 2009), e Maillard, Flamand 2010 (come a n. 25), 1-214.

27 Sanchi 2018 (come a n. 3), 10.

${ }^{28}$ Sanchi 2006 (come a n. 4), 246.

${ }^{29}$ Edito in L. Petit, X.A. Sideridès, M. Jugie, Oeuvres complètes de Georges (Gennadios) Scholarios, vol. 3 (Paris, 1930), 453-458. Si veda anche M. des Portes, "Le Bref exposé de la foi chrétienne du théologien Gennadios Scholarios. Présentation et traduction", Réforme, Humanisme, Renaissance 78 (2014), 246-258. 


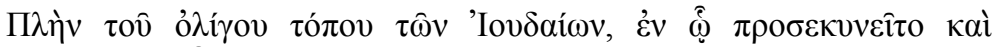

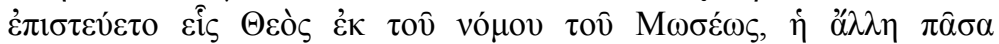

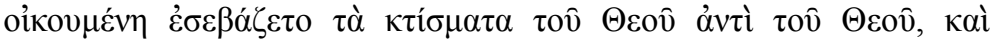

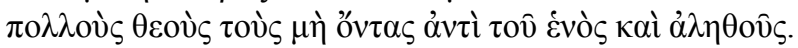

Eccetto la piccola regione della Giudea, in cui si adorava e si credeva in un unico Dio secondo la legge di Mosè, tutto il restante mondo venerava le creature di Dio anziché Dio, e molti dei che in realtà non esistono anziché l'unico e vero Dio.

Quest'opera contiene la professione di fede del patriarca costantinopolitano, scandita dall'anafora del verbo $\pi 1 \sigma \tau \varepsilon v ́ o \mu \varepsilon v$, "crediamo". In alcuni

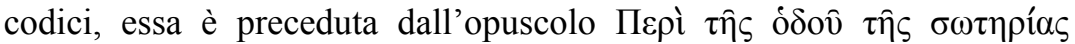
$\alpha \nu \theta \rho \omega ́ \pi \omega \nu$ ("Sulla via della salvezza per gli uomini”), conosciuto anche come Confessio fidei prior o De via salutis, che, nonostante nei testimoni sia attribuito a Scolario, è ritenuto spurio dalla critica. ${ }^{30}$

Analizzando i manoscritti contenenti sia il De via salutis sia la Confessio fidei posterior, ${ }^{31}$ notiamo come quasi tutti ${ }^{32}$ siano stati copiati interamente o parzialmente dal maestro di Budé Giorgio Ermonimo: sono i codici Wien, Österreichische Nationalbibliothek, ms. Suppl. Gr. 73; Paris, Bibliothèque nationale de France, ms. Gr. 1298; Firenze, Biblioteca Medicea Laurenziana, ms. Plut. 7.34; Kraków, Biblioteka Jagiellońska, ms. 9964. ${ }^{33}$ Siccome questi sono anche i più antichi manoscritti del De via salutis, Martin Jugie ha ipotizzato che sia stato proprio Ermonimo a comporlo, attribuirlo al patriarca di Costantinopoli e ad abbinarlo alla Confessio fidei posterior autentica-

${ }^{30} \mathrm{Si}$ veda da ultimo G. Karamanolis, "Form and Content in the Dialogues of Gennadios Scholarios", in A. Cameron, N. Gaul (ed.), Dialogues and Debates from Late Antiquity to Late Byzantium (London - New York, NY, 2017), 237-251 (a 239-240, 248). L'edizione più recente del De via salutis si legge in J.P. Migne (ed.), Patrologia Graeca, vol. 160 (Lutetiae Parisiorum, 1866), 434-452.

${ }^{31}$ Il sito web Pinakes registra quindici manoscritti con il De via salutis (https://pinakes.irht.cnrs.fr/notices/oeuvre/1636/) e settantadue testimoni della Confessio posterior (https://pinakes.irht.cnrs.fr/notices/oeuvre/1635/).

32 Pinakes ne registra quattro, ovvero tre manoscritti copiati da Ermonimo (Vind. suppl. gr. 73; Par. gr. 1298; Laur. Plut. 7.34) e uno atonita (Athos, Mone Laura, ms. K 12) datato 1665 .

${ }^{33}$ Le descrizioni di questi manoscritti si leggono, nell'ordine, in Kalatzi 2009 (come a n. 26), 179-181, 237-238, 271-272, 324-326; e Maillard, Flamand 2010 (come a n. 25), 26-30, 75-76, 131-132, 158-162. 
mente scolariana. ${ }^{34}$ Ermonimo curò anche una traduzione latina di Confessio prior e posterior. ${ }^{35}$

In base alle indagini condotte da Kalatzi, Maillard e Flamand, ${ }^{36}$ nessuno dei quattro manoscritti greci citati reca note o qualsivoglia traccia dell'attività erudita di Budé. Egli però sicuramente trasse la citazione di Scolario da un codice, giacché l'editio princeps di Confessio prior e posterior è datata $1530,{ }^{37}$ mentre la citazione si legge già nell'edizione del 1529 dei Commentarii. ${ }^{38}$

In assenza di note di Budé nei manoscritti greci copiati da Ermonimo, quindi, non è neppure sicuro che egli si sia servito di uno dei codici del suo maestro. Sarebbe perciò necessario controllare tutti i testimoni della Confessio posterior $^{39}$ per vedere se qualcuno di essi sia stato annotato da Budé. ${ }^{40}$

Per concludere, dato il grande interesse di Ermonimo per quest'opera scolariana, è plausibile che Budé abbia potuto leggerla o ne sia venuto a conoscenza grazie al suo maestro, ma ad ora risulta impossibile stabilire quale sia il manoscritto da cui Budé trasse la citazione della Confessio posterior.

The Warburg Institute, London gianmario.cattaneo@sas.ac.uk

${ }^{34}$ Petit, Sideridès, Jugie 1930 (come a n. 30), xxxi-xxxiv. Si veda a proposito anche Kalatzi 2009 (come a n. 26), 105-106.

${ }^{35}$ Su questa versione si rimanda a Kalatzi 2009 (come a n. 26), 104-106.

${ }^{36} \mathrm{Si}$ veda la n. 33. Ho ricontrollato le riproduzioni digitali dei manoscritti Par. gr. 1298 e Laur. Plut. 7.34, confermando l'assenza della mano di Budé in essi.

37 Gennadius Scholarius, De syncaeritate Christianae fidei dialogus (Vindobonae, 1530 [USTC 659319]). La Confessio posterior si legge ai f. o $1 \mathrm{v}-\mathrm{t} 4 \mathrm{v}$.

38 Budé 1529 (come a n. 9), 580.

${ }^{39} \mathrm{Si}$ veda la n. 31.

${ }^{40}$ Tra i manoscritti prodotti tra il Quattrocento e l'inizio del Cinquecento, ho controllato, senza trovare traccia della mano di Budé, su riproduzione digitale i codici Città del Vaticano, Biblioteca Apostolica Vaticana, ms. Pal. gr. 297; ms. Reg. gr. 43; London, British Library, ms. Harley 5734; München, Bayerische Staatsbibliothek, ms. Gr. 490; Paris, Bibliothèque nationale de France, ms. Gr. 1293, 1294, 2135, 2551. 